\title{
Method to Predict Reagents in Iridium-Based Photoredox Catalysis
}

\author{
Antoine Juneau, Taylor O. Hope, Jason Malenfant, Mihai Mesko, Jacob McNeill and Mathieu Frenette* \\ Department of Chemistry and NanoQAM, Université du Québec à Montréal, Case Postale 8888, Succursale Centre- \\ Ville, Montréal, Québec H3C 3P8, Canada
}

\begin{abstract}
Visible-light photoredox catalysts with oxidizing excited states have been broadly applied in organic synthesis. Following photon absorption by the photocatalyst, electron transfer from an organic reagent is the most common mechanistic outcome for this class of reaction. Reduction potentials for organic reagents are therefore useful to predict reactivity and DFT proved to be useful as a predictive tool in this regard. Due to the complex mechanisms that follow electron transfer, kinetics play a crucial role in the success of photoredox reactions. We extend the predictive tools of DFT to estimate the electron transfer rates between an excited photocatalyst and various organic substrates. To calibrate our model, 49 electron transfer rate constants were experimentally measured in acetonitrile for the catalyst $\operatorname{Ir}\left[\mathrm{dF}\left(\mathrm{CF}_{3}\right) \mathrm{ppy}\right]_{2}(\mathrm{dtbpy})^{+}$. The rate constants, $\mathrm{k}_{q}$, gave a clear predictive trend when compared to calculated ionization energies in "frozen solvent", which was a better predictor than standard reduction potentials in our case. The calculated $\mathrm{k}_{q}$ gave an average error of $17 \%$ for $\log \left(\mathrm{k}_{\mathrm{q}}\right)$ values between 4 and 11. This simple method can predict the reactivity of hundreds of reagents in silico. Notably, the calculations offered unexpected insight that we could translate into success for the $\mathrm{C}-\mathrm{H}$ activation of acetylacetone as a proof-of-concept.
\end{abstract}

\section{Introduction}

Photoredox catalysis is an emerging field of applied photochemistry enabled by bright and efficient visible light sources. Organic synthesis, in particular, benefited from novel light-initiated transformations that range from $\mathrm{C}-\mathrm{C}, 1,2$ $\mathrm{C}-\mathrm{N},{ }^{3,4} \mathrm{C}-\mathrm{O}^{5}$ and $\mathrm{C}-\mathrm{S}^{6}$ bond forming reactions (Scheme 1 ). Since many photoredox reactions are radical-based mechanisms, these reactions can tolerate functional groups that usually interfere with standard nucleophile/electrophile chemistry, ${ }^{7}$ e.g., alcohols and ketones need no protection/deprotection steps for many photoredox transformations. Photocatalysis has another major advantage over traditional methods: the reactions conditions are mild and often do not require harsh reagents or high temperatures. ${ }^{8}$ Several reactions cited as examples in references 1-6 are catalyzed by the same iridium photocatalyst: $\operatorname{Ir}\left[\mathrm{dF}\left(\mathrm{CF}_{3}\right) \mathrm{ppy}\right]_{2}(\mathrm{dtbpy})+\mathrm{PF}_{6}-\left(\mathbf{I r}^{\mathrm{III}} \mathbf{L}\right)$. Ir ${ }^{\mathrm{III}} \mathbf{L}_{\mathbf{n}}$ has a long-lived and strongly oxidizing excited state $\left(\tau_{0}=2.3 \mu \mathrm{s}\right.$ and $\mathrm{E}_{1 / 2}{ }^{\mathrm{red}}\left({ }^{*} \mathbf{I r}^{\mathrm{IV}} \mathbf{L n}^{{ }^{-}} / \mathbf{I r}^{\mathbf{I I I}} \mathbf{L n}_{\mathbf{0}^{-}}{ }^{-}\right)=+1.21 \mathrm{~V}$ vs SCE in MeCN $)^{\cdot{ }^{9}}$ While iridium is a rare and expensive metal and several catalysts have been proposed as alternatives, ${ }^{9,10}$ iridium is also a crucial component in many reactions to achieve high yields beyond the milligram scale.

Iridium-based photoredox catalysis often undergoes interestingly complex multi-step mechanisms that are still a matter of discussion in the literature. The success of an overall reaction is dictated by the kinetics of several competing reactions that are difficult to predict. To better foresee reaction outcomes and to uncover novel reactivity, it is highly desirable to apply quantitative predictive models to these reactions. At this stage, however, ab initio calculations of reaction rates, in particular, electron transfer reaction rates from excited states, are not trivial to conduct and interpret. With the goal of helping the synthetic community discover novel reactants in photoredox catalysis, we set out to conceive a simple computational method to obtain reliable electron transfer rates that are calibrated with experimentally measured rate constants.
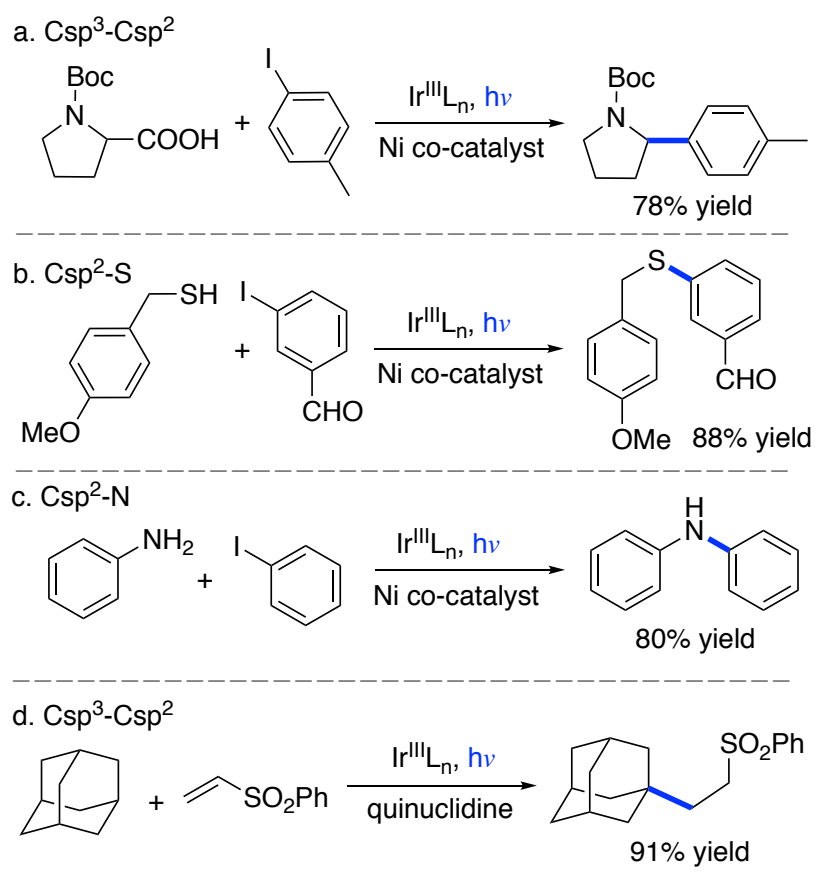

Scheme 1. Photoredox reactions using iridium-based photocatalysts to carry out (a) C-C cross coupling via carboxylate oxidation ${ }^{1}$, (b) C-S cross coupling via thiol oxidation ${ }^{6}$, (c) C-N cross coupling via aniline oxidation ${ }^{3}$, and (d) hydrogen selective $\mathrm{C}-\mathrm{H}$ functionalization of adamantanes. ${ }^{2}$ 
We carefully measured 49 rate constants for electron transfer reactions between the excited state of I IIII ${ }^{\mathrm{In}}$ and a wideranging series of electron-donating quenchers, $\mathbf{Q}$. This data allowed us to fine-tune our predictive computational model, while retaining an ease of use at a small computational cost. Indeed, most photoredox catalysis mechanisms that begin with light absorption by IrIII $\mathbf{L}_{\mathbf{n}}$ are followed by an electrontransfer step from a substrate, $\mathbf{Q}$ (Scheme 2). As mentioned earlier, the photocatalyst excited state is long-lived, due to its triplet electronic configuration, and behaves as a strong oxidizing agent. The excited state ${ }^{*} \mathbf{I r}^{\mathrm{III}} \mathbf{L}_{\mathbf{n}}$ is better described

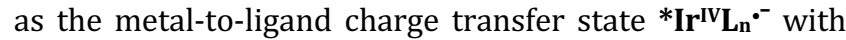
hole at the metal-centered $t_{2 g}$ orbital and an extra electron in the ligand $\pi^{*}$ system (Scheme 2). The reaction between ${ }^{*} \mathbf{I r}^{\mathrm{IV}} \mathbf{L}_{\mathbf{n}}{ }^{-}$and $\mathbf{Q}$ has a rate constant, $\mathrm{k}_{q}$, that can be measured by the well-established Stern-Volmer kinetic method (Figure 1). Since IrIII $\mathbf{L n}_{\mathbf{n}}$ has a known excited state lifetime with strong phosphorescence, $\mathbf{Q}$ can be introduced to the system to establish a competitive kinetic between emission and electron transfer. As more quencher is added, the emission intensity will be reduced by a ratio given by the SternVolmer equation (eq. 1).

$$
\left.\frac{I_{0}}{I}=1+k_{q} \tau_{0}[Q] \quad \text { (eq. } 1\right)
$$

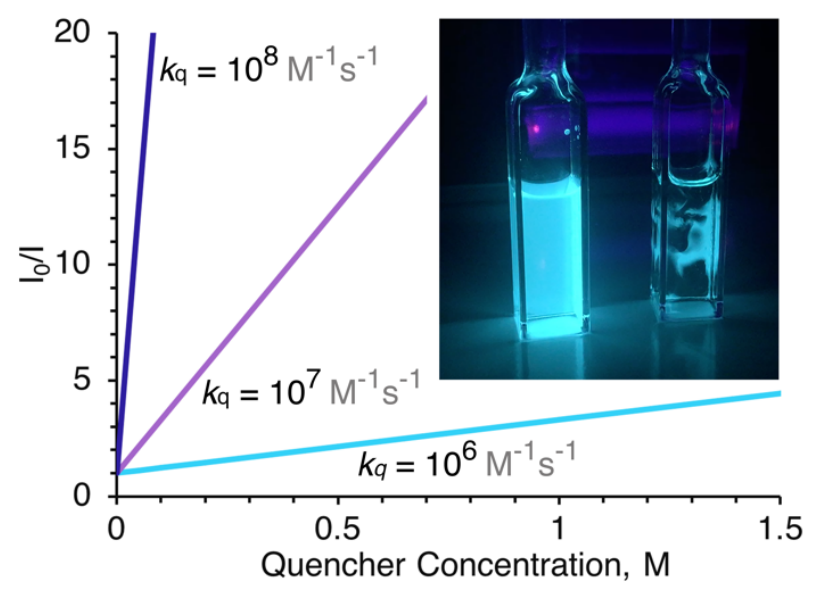

Figure 1. Idealized Stern-Volmer plot with fast (dark blue), medium (purple) and slow (pale blue) quenchers. Inset: Ir'II $\mathbf{L}_{\mathbf{n}}$ in degassed acetonitrile under UV-light excitation before (left) and immediately following (right) addition of an amine quencher (DABCO was used for this picture) to illustrate the emission quenching by the electron transfer reaction. Experimental quenching data and the video from which the image is taken are available in the SI.

The products of the excited-state electron transfer reaction, Ir III $^{\text {Inn }}{ }^{-}$and $\mathbf{Q}^{\bullet+}$, will go on to further reduction, oxidation, proton-transfer or radical-addition steps. It should be noted that a subset of research with Ir' ${ }^{\text {III }} \mathbf{L}_{\mathbf{n}}$ could potentially include reduction steps or energy transfer mechanisms; ${ }^{11}$ our model focuses on the more common single electron oxidation of $\mathbf{Q}$ to form $\mathbf{Q}^{\cdot+}$.

While the overwhelming list of quenchers in the literature are alkyl amines, there are many other classes of molecules that can react with ${ }^{*} \mathbf{I r}^{\mathbf{I V}} \mathbf{L n}^{{ }^{-}}$. We set out to quantify their reactivity with Ir'III $\mathbf{L}_{\mathbf{n}}$ excited states and use this data to predict novel reactivity that will be useful in reaction discovery by the synthetic community at large.

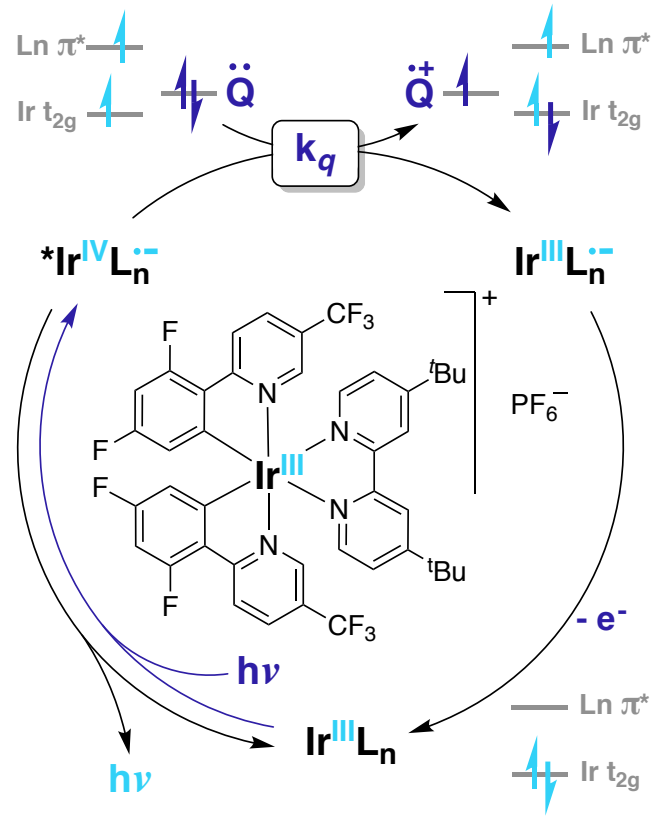

Scheme 2. Typical photoredox catalytic cycle for $\mathbf{I r}^{\mathrm{III}} \mathbf{L}_{\mathbf{n}}$ ( $\left.\operatorname{Ir}\left[\mathrm{dF}\left(\mathrm{CF}_{3}\right) \text { ppy }\right]_{2}(\mathrm{dtbbpy})+\mathrm{PF}_{6}{ }^{-}\right)$. Absorption of a photon by Ir ${ }^{\text {III }} \mathbf{L n}$ eventually creates a triplet excited state, ${ }^{*} \mathbf{I}^{\mathbf{I V}} \mathbf{L n}^{{ }^{-}}$, that can emit a photon or oxidize a quencher, $Q$, with rate constant $\mathrm{k}_{q}$. The reduced iridium species, I $\mathbf{I}^{\mathrm{III}} \mathbf{L}_{\mathbf{n}}{ }^{-}$(often called IrII) can donate an electron to complete the catalytic cycle.

\section{Results and Discussion}

The precision of Density Functional Theory (DFT) to determine the oxidation and reduction potentials of organic compounds ${ }^{12}$ motivated us to apply this tool to the kinetics of SET with the Ir'II'Ln photocatalyst. The study was deliberately carried out on various molecules (phenols, trifluoroborate salts, amines, phosphine, nickel complex) in order to obtain a coarse but general model. To our knowledge, similar studies have focused on a restricted type of aromatic compound with different substitution in the ortho meta or para position such as arylamines, or alkoxybenzene.13,14, 15 More specifically, the objective was to evaluate the relationship between the quencher rate constants $\left(\mathrm{k}_{q}\right)$ and the emission of * Ir $^{\mathbf{I V}} \mathbf{L n}{ }^{-}$by different quenchers (Q) for the SET.

In our model, the energy contribution from the half reduction reaction of ${ }^{*} \mathbf{I r}^{\mathrm{I}} \mathbf{L}_{\mathbf{n}}{ }^{-}$is assumed to be constant. For bulky organometallic photocatalysts, outer sphere electron transfer mechanism are the most likely; there should be no specific molecular interactions between the photocatalyst and the quencher. We can therefore assume that the geometric and electronic structure of the reduced and oxidized quencher to be independent of the photocatalyst, which allows the assumption of geometric and electronic structures to be close to the ground state.

Stern-Volmer kinetic analysis, measures the reactivity of activated species such as the excited state of iridium. With this approach, the $k_{q}$ of ${ }^{*} \mathbf{I r}^{\mathrm{IV}} \mathbf{L}_{\mathbf{n}}{ }^{-}$-were measured for a total of 49 molecules: 20 amines (Figure 2), 15 phenols (Figure 3) and 14 various molecules (Figure 4). These include various phosphines, alkanes, thiol, trifluoroborate salts, etc. ${ }^{16,17}$ Results obtained for these 49 rate constants between ${ }^{*} \mathbf{I r}^{\mathrm{IV}} \mathbf{L}_{\mathbf{n}}{ }^{-}$ and $\mathbf{Q}$ are summarized in Figures 2, 3 and 4 below. All quenching data is included in the supporting information. 


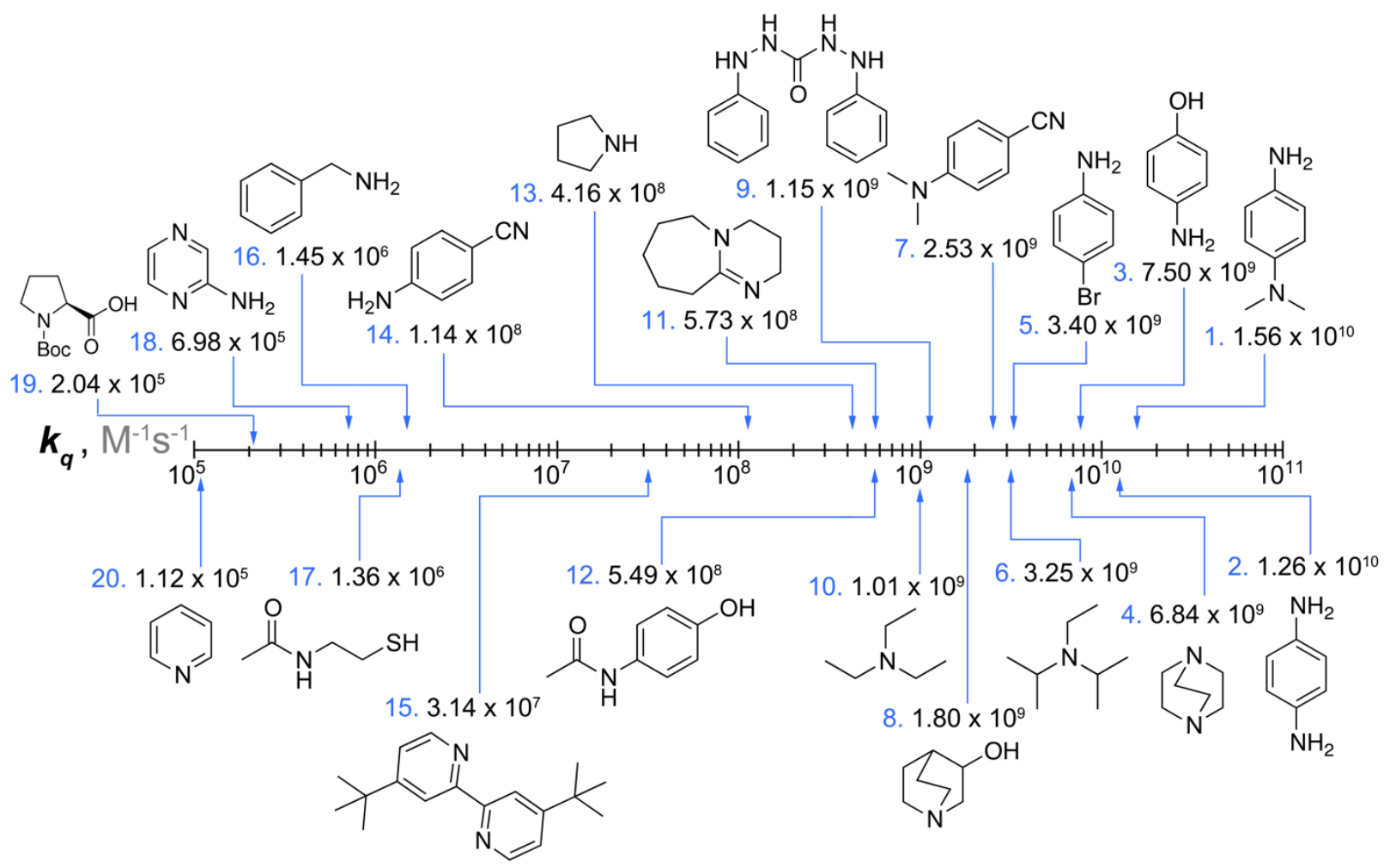

Figure 2. Bimolecular quenching rate constants $\left(\mathrm{k}_{q}\right)$ for the reaction of ${ }^{*} \operatorname{Ir}^{\mathbf{I V}} \mathbf{L}_{\mathbf{n}}{ }^{{ }^{-}}$with various amines and nitrogen-containing molecules in acetonitrile. See supporting information for raw quenching data.

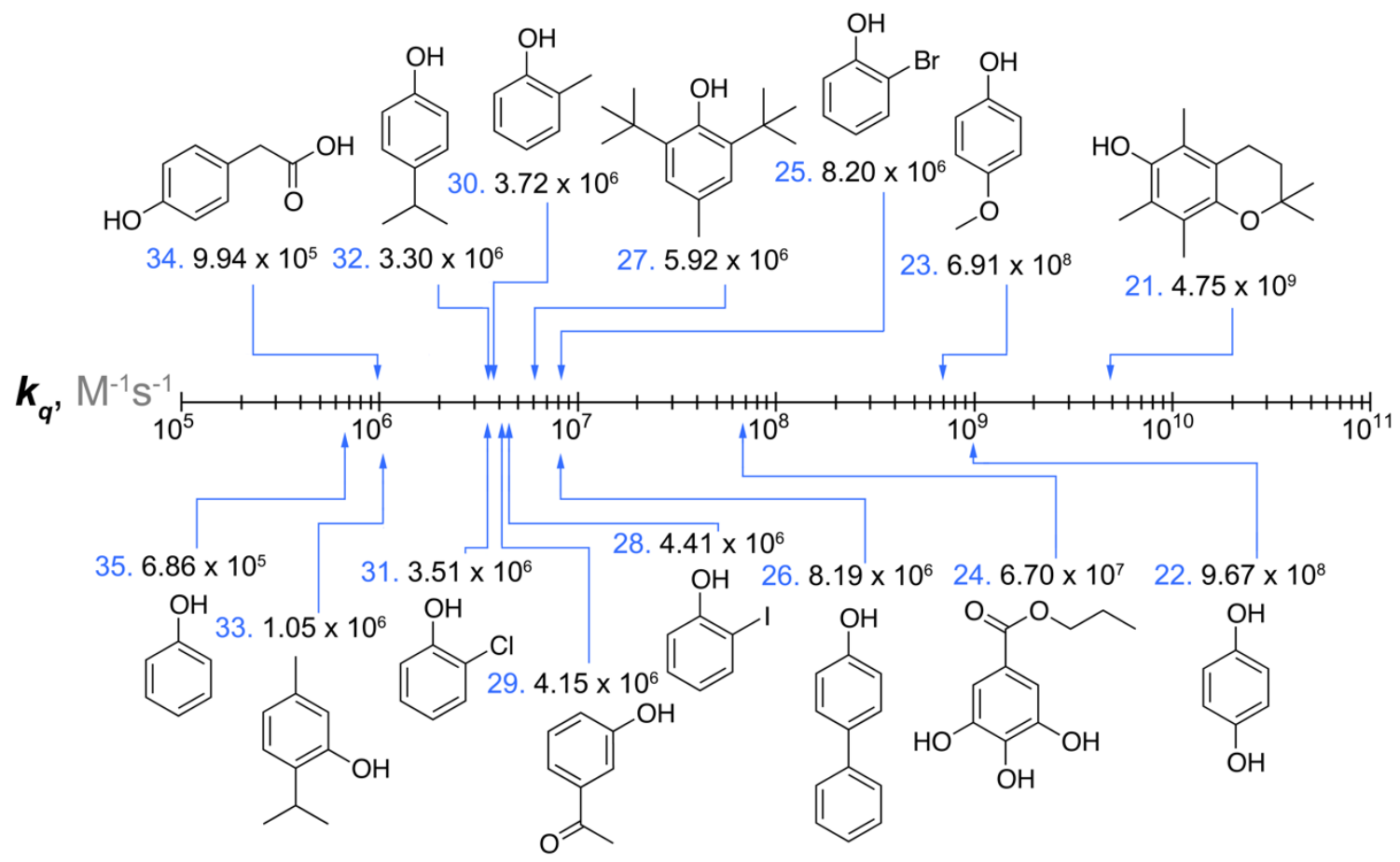

Figure 3. Bimolecular quenching rate constants $\left(\mathrm{k}_{q}\right)$ for the reaction of ${ }^{*} \mathbf{I r}^{\mathbf{I V}} \mathbf{L}_{\mathbf{n}}{ }^{\cdot-}$ with various phenolic compounds in acetonitrile. See supporting information for raw quenching data. 

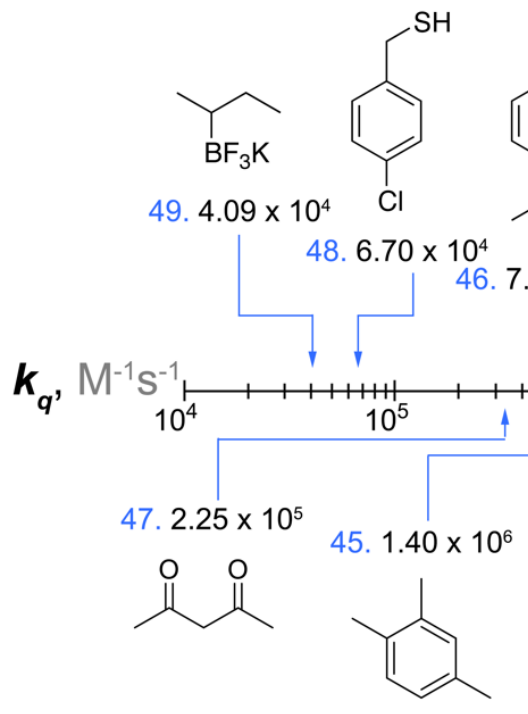<smiles>COc1ccc(CS)cc1</smiles><smiles>O=C(OCCOCCOCCOCCO)c1ccccc1</smiles>

43. $1.20 \times 10^{7} \quad \mathrm{BF}_{3} \mathrm{~K}$

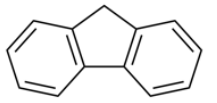

41. $1.00 \times 10^{8}$

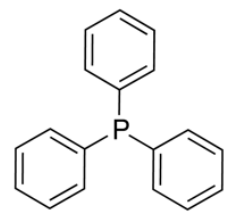

39. $2.82 \times 10^{8}$
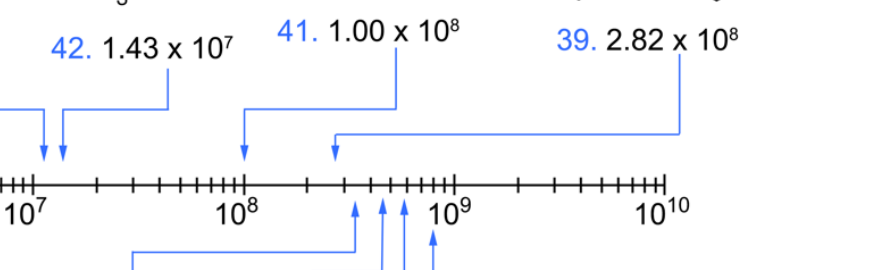
$0^{10}$

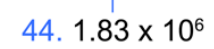

40. $2.65 \times 10^{8}$<smiles>Cc1c(C)c(C)c(C)c(C)c1C</smiles><smiles>O=C([O-])C1CCCN1C(=O)O</smiles><smiles>CC(=O)OC(C)=O</smiles>

Figure 4. Bimolecular quenching rate constants $\left(\mathrm{k}_{q}\right)$ for the reaction of ${ }^{*} \operatorname{Ir}^{\mathbf{I V}} \mathbf{L}_{\mathbf{n}}{ }^{-}$with various compounds in acetonitrile. The quenching rate constants for compounds $\mathbf{4 4 , 4 5}$ and $\mathbf{4 9}$ are lower than the assigned value due to poor linearity in the quenching data. See supporting information for raw quenching data.

Several trends in reactivity could be rationalized based on the quencher structure; however, the goal here is to gather experimental rate constants in order to develop and validate a predictive model for photoredox reaction reagents.

\section{Predicting Reactants in Photoredox Catalysis}

We compared the experimental rate constants $\mathrm{k}_{q}$ with DFT calculations according to two measurements from the electron transfer process: Gibb's free energy $\left(\Delta \mathrm{G}_{\mathrm{ET}}\right)$ and ionization energy (IE) (Scheme 3). The Gibb's free energy calculations are related to the reduction potentials ( $\mathrm{E}^{\circ}$ ) used by most chemists in the field since $\Delta \mathrm{G}^{\mathrm{o}}=-\mathrm{nFE}$. We found the $\Delta \mathrm{G}$ measurements problematic in several instances to predict rates (see SI). For some classes of reagents, there can be a significant rearrangement of the radical cation $\mathbf{Q}^{\bullet+}$ structure. For example, electron loss will cause some bonds to be broken, some metal complexes to change spin states, and often, significant changes to occur in dihedral angles. These geometric rearrangements following oxidation of $\mathbf{Q}$ should not be taken into account in our empirical model-secondary structural relaxation will occur after the electron transfer has occurred and should play a less important role in the energy barrier for the actual electron transfer step.

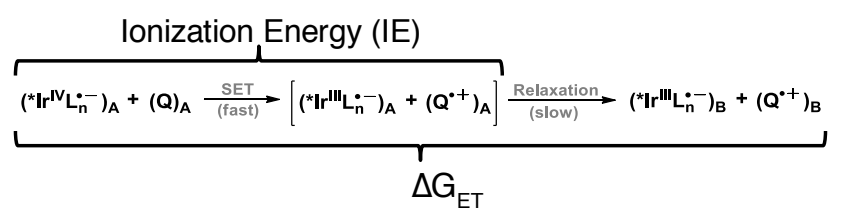

Scheme 3. Detailed processes surrounding an electron transfer reaction between an excited photocatalyst $\left({ }^{*} \mathbf{I r}^{\mathbf{I V}} \mathbf{L}^{{ }^{-}}\right)$and a quencher $\mathbf{Q}$. In this representation, subscripts A denotes the reactant's ground state geometries and subscript $B$ denotes the product's final geometries after relaxation.
Instead of correlating measured $\mathrm{k}_{q}$ with $\Delta \mathrm{G}_{\mathrm{ET}}$ (or $\mathrm{E}^{\mathrm{o}}$ ), we opted to correlate the experimental data with calculations for the ionization energy (IE). Geometric reorganization of the $\mathbf{Q}^{\cdot+}$ structure is not taken into account in these calculations and we found this approach to have two advantages: 1 ) it more closely mimics the fast electron transfer that occurs faster than atomic motion and 2) it is computationally less time consuming since only one geometry optimization is needed for $\mathbf{Q}$ before electron transfer has occurred.

Different levels of theory were tested to obtain IE for all quenchers. Three functionals that have been tested (B3LYP, wB97XD and M06-2x). The functional B3LYP (Becke, 3-parameter, Lee-Yang-Parr) is good for a wide range of applications (transition metals, NMR, energy barrier, among others). It is the most widely used in literature and was a good starting point. The M06-2x functional (Minnesota functional from 2006) was used because it performed better than B3LYP for ionization potentials in the aqueous phase without being as demanding as methods such as CCSD(T). ${ }^{18}$ Finally, wB97XD ${ }^{19}$ better estimates vertical ionization energy than M06-2x and B3LYP for small organic molecules. ${ }^{20}$ As predicted, the geometries for all three functionals optimized in a $\operatorname{CPCM}(\mathrm{MeCN})$ model using the basis set $6-311+g(2 d, 2 p)$ converged to similar structures (see SI). Empirically, however, we obtained a better fit for $\mathrm{k}_{q}$ vs IE using the B3LYP level of theory. Our calculations also employed the "nonEq" solvent approximation as implemented in Gaussian09.21 For these calculations, the CPCM solvent is first optimized for the geometrically optimized neutral quencher $\mathbf{Q}$. In a second calculation, the solvent's slow component of the dielectric constant is kept constant for the geometrically frozen calculation of $\mathbf{Q}^{\bullet+}$. This approach was taken to simulate the fact that electron transfer occurs faster than solvent reorganization. The ionization energy is taken as the difference between the lowest SCF energies for $\mathbf{Q}$ and $\mathbf{Q}{ }^{\bullet+}$. It 
would be impossible to calculate a proper $\Delta \mathrm{G}$ for this hypothetical electron transfer since the geometry of $\mathbf{Q}^{\bullet+}$ is not optimized and thermodynamic calculations in Gaussian (and most quantum chemistry packages) assume a harmonic oscillator model at a local minimum (or maximum) for each vibrational mode.

Figure 5 shows the correlation between the measured quenching rate constants, as $\log \left(\mathrm{k}_{q}\right)$, versus calculated ionization energies in $\mathrm{eV}$.

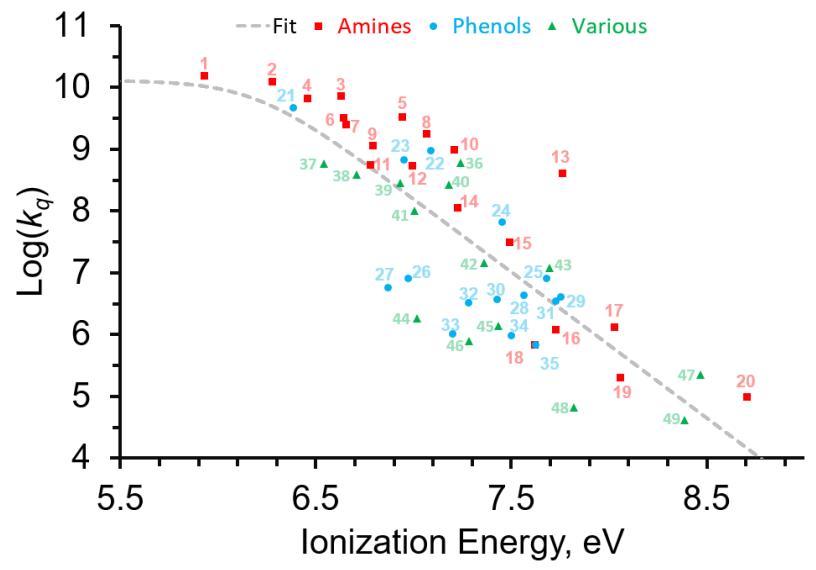

Figure 5. Predictive Model for Reactants in IridiumBased Photoredox Catalysis. Relationship between the experimentally measured $\log \left(\mathrm{k}_{q}\right)$ for the reaction of $* \mathbf{I r}^{\mathbf{I}}$ $\mathbf{v}^{\mathbf{L n}^{*}}{ }^{-}$with quenchers and the quencher's ionization energy as calculated by DFT (B3LYP/6-311+G(2d,2p) //CPCM(MeCN) with "frozen solvent", see SI). Data for amines and nitrogen-containing compounds (red squares), phenols (blue circles), various molecules (green triangles) were fit with a trend according to the RehmWeller model (dashed gray line). Experimental and theoretical values were obtained/calculated in acetonitrile.

Overall, our model can predict whether a molecule is a potential reagent for reaction with ${ }^{*} \mathbf{I}^{\mathbf{I V}} \mathbf{L}_{\mathbf{n}} \mathbf{0}^{-}$. Some outliers, such as 27, with tert-butyl side chains on the phenol or $\mathbf{3 3}$ with isopropyl side chains, react more slowly than the model would predict. The clear culprit for this discrepancy is steric hinderance near the phenol moiety since our calculations do not account for steric hinderance.

The trend presented in gray in Figure 5 was obtained according to the equation below using the diffusion constant in acetonitrile of $2.9 \times 10^{10} \mathrm{M}^{-1} \mathrm{~S}^{-1}:^{15}$

$\mathrm{k}_{q}=\frac{\mathrm{k}_{\mathrm{ET}} \times \mathrm{k}_{\text {diff }}}{\mathrm{k}_{\mathrm{ET}}+\mathrm{k}_{\text {diff }}}=\frac{\mathrm{k}_{\mathrm{ET}} \times\left(2.9 \times 10^{10} \mathrm{M}^{-1} \mathrm{~s}^{-1}\right)}{\mathrm{k}_{\mathrm{ET}}+\left(2.9 \times 10^{10} \mathrm{M}^{-1} \mathrm{~s}^{-1}\right)}$

For which $k_{q}$ was obtained assuming that the logarithm of the speed constant of electron transfer $\left(\mathrm{k}_{\mathrm{ET}}\right)$ was proportional to the ionization energy determined by DFT. Note that $1 \mathrm{eV}$ is equal to 0.03675 Hartree.

$\log \left(\mathrm{k}_{\mathrm{ET}}\right.$ in $\left.\mathrm{M}^{-1} \mathrm{~s}^{-1}\right)=-2.363 \mathrm{eV}^{-1} \mathrm{x}(\mathrm{IE}$ in eV) +24.73 (eq. 3)

Using this correlation, one can conduct a simple DFT calculation on a potential quencher to obtain IE and this value can be converted into $\mathrm{k}_{\mathrm{ET}}$ and $\mathrm{k}_{q}$. In cases where $\mathrm{k}_{\mathrm{ET}}$ is lower than $\sim 10^{8} \mathrm{M}^{-1} \mathrm{~s}^{-1}, \mathrm{kET}_{\mathrm{ET}}$ can be assumed equal to $\mathrm{k}_{q}$. The correction is needed when $\mathrm{k}_{\mathrm{ET}}$ estimates are close to or above the maximum diffusion rate-the rate constants cannot be faster than diffusion in the assumed model for electron transfer.

This correlation gave $\log \left(\mathrm{k}_{q}\right)$ values with $\sim 17 \%$ error between theory and experiment in the range between 4 and 11 . The phenol series were particularly problematic, and this was expected since the electron transfer for several phenols will face steric hinderance. The largest error in our model is attributed to 2,6-di-tert-butyl-4-methylphenol (27), also known as BHT. Experimentally, $\log \left(\mathrm{k}_{q}\right)$ for 27 was measured as 6.8 while the calculated IE predicted a $\log \left(\mathrm{k}_{q}\right)$ of 8.5 . The large discrepancy is presumably due to steric hindrance that is not considered in our computer model. Overall, the predicted rates were in very good agreement with electron-donating quenchers without steric hinderance. Amines and anions, in particular, reacted with rate constants that closely matched calculations.

If more than one quencher is in solution $(A, B, \ldots)$, our model can predict rate constant for each quencher $\left(\mathrm{k}_{q}{ }^{\mathrm{A}}, \mathrm{k}_{q}{ }^{\mathrm{B}}\right.$, ...). With this information and the photocatalyst lifetime $(t)$, we can estimate the percentage of excited states that react with a particular reagent using the equation 4 :

$$
\% \text { quenching by } \mathrm{A}=\frac{100 \times \mathrm{k}_{q}{ }^{\mathrm{A}}[\mathrm{A}]}{\tau^{-1}+\mathrm{k}_{q}^{\mathrm{A}}[\mathrm{A}]+\mathrm{k}_{q}^{\mathrm{B}}[\mathrm{B}]+\ldots}
$$

In the equation, values for $[\mathrm{A}]$ and $[\mathrm{B}]$ must be in mol/L and $\tau$ in seconds. If oxygen is present in the medium, it will be necessary to account for quenching by oxygen with an additional $\mathrm{k}_{q}{ }^{02}\left[\mathrm{O}_{2}\right]$ term. We experimentally measured $\mathrm{k}_{q}{ }^{02}$ with $\mathrm{O}_{2}$ to be $2.71 \times 10^{9} \mathrm{M}^{-1} \mathrm{~s}^{-1}$ and the $\mathrm{O}_{2}$ concentration is $2.42 \mathrm{mM}$ in air-saturated acetonitrile. ${ }^{6}$

\section{Discovering photoredox reagents}

Our predictive model can be used to estimate the quenching rate constant $\mathrm{k}_{q}$ of any molecule that behaves as an electron-donating quencher to Ir'III Ln. Limited only by imagination and computational resources, we calculated "frozen solvent" ionization energies (IE) for 100+ small molecules. Within the theoretical results, some quenchers gave surprisingly high predicted $\mathrm{k}_{q}$ values. For example, the potassium salt of 2,4-pentanedione anion (38) and neutral fluorene (49) were predicted to react with $\log \left(\mathrm{k}_{q}\right)$ of 8.6 and 8.0, respectively. Following the calculations, we experimentally measured the quenching rate constants to verify our predictions. Satisfyingly, we found that the predicted rate constant for these compounds were within 3 and $2 \%$ to the experimentally measured $\log \left(\mathrm{k}_{q}\right)$.

As a proof of concept, we attempted to develop a reaction that will take advantage of the reactivity of 2,4-pentanedione anion (38) and fluorene (49). While fluorene does react with ${ }^{*} \mathbf{I r}^{\mathbf{I V}} \mathbf{L n}^{-{ }^{-}}$via SET, we were not able to translate this reactivity into a useful synthetic transformation. The radical cation derived from fluorene will rapidly deprotonate ( $\mathrm{pKa}$ fluorene $\cdot+=-17)^{22}$ to yield a particularly stable radical: fluorenyl radical. The fluorenyl radical is calculated to give endergonic addition reactions with most typical radical acceptors and experimental attempts at isolating addition products were not productive (data not shown).

We were successful, however, in translating 2,4-pentanedione anion (38) reactivity into a proper photoredox transformation (Scheme 4). 


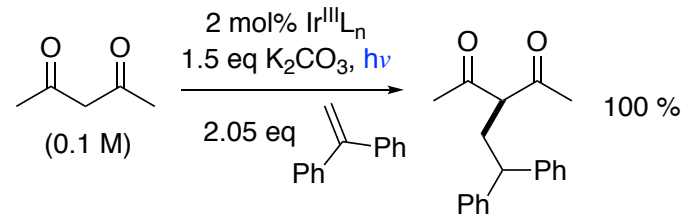

Proposed mechanism

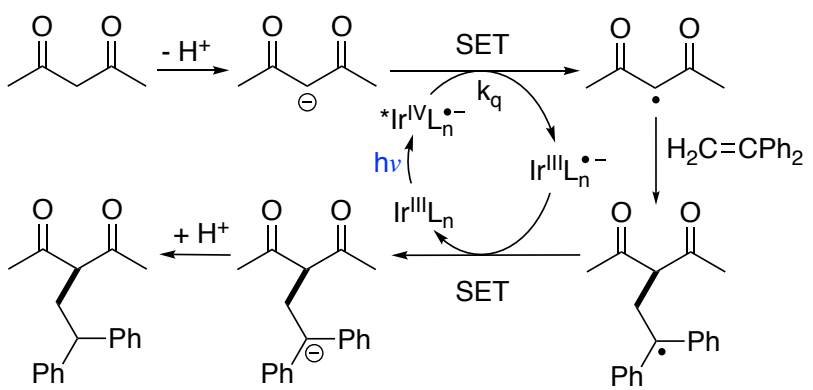

Scheme 4. Our theoretical model predicted a rapid reaction of deprotonated 2,4-pentanedione with ${ }^{*} \mathbf{I r}^{\mathbf{I V}} \mathbf{L}_{\mathbf{n}}{ }^{-}$to yield the 2,4-pentanedienyl radical. Under the reaction conditions listed, the radical can add to diphenylethylene to form a carbon-carbon bond in 100\% yield (measured by GC-MS on milligram scale). A proposed mechanism is also shown.
The goal of this work was to predict novel reagents that could be used in photoredox catalysis. With this in mind, we present a list of molecules for which we predicted electron transfer rate constants (Figure 6). These rate constants were not measured experimentally, nor was their reactivity verified by synthesis, however, we believe our model can be useful as a guide to discover novel reactivity. A surprise, at least to us, was the rapid rates seen for carbanions. As seen for the 2,4-pentanedione anion, we calculate rate constants that approach diffusion control for anions 52 and 54. Some reagents were also notably slower: compound $\mathbf{7 2}$ is predicted to react very slowly as a neutral reagent, but will be quicly oxidized by ${ }^{*} \mathbf{I r}^{\mathbf{I V}} \mathbf{L n}^{{ }^{*-}}$ as an anion (50). Another standout example is the product formed by the addition of hydroxide to $\mathrm{B}_{2} \mathrm{Pin}_{2}(73)$ to form $\mathrm{B}_{2} \mathrm{Pin}_{2} \mathrm{OH}^{-}$which makes an excellent reducing agent (55).

So far, all the rate constants were measured and calculated for one photocatalyst, $\operatorname{Ir}\left[\mathrm{dF}\left(\mathrm{CF}_{3}\right) \mathrm{ppy}\right]_{2}(\mathrm{dtbpy})^{+} \mathrm{PF}_{6}$. We intend to generalize the calculated rate constants for any oxidizing photocatalyst that operates via outer sphere electron transfer.

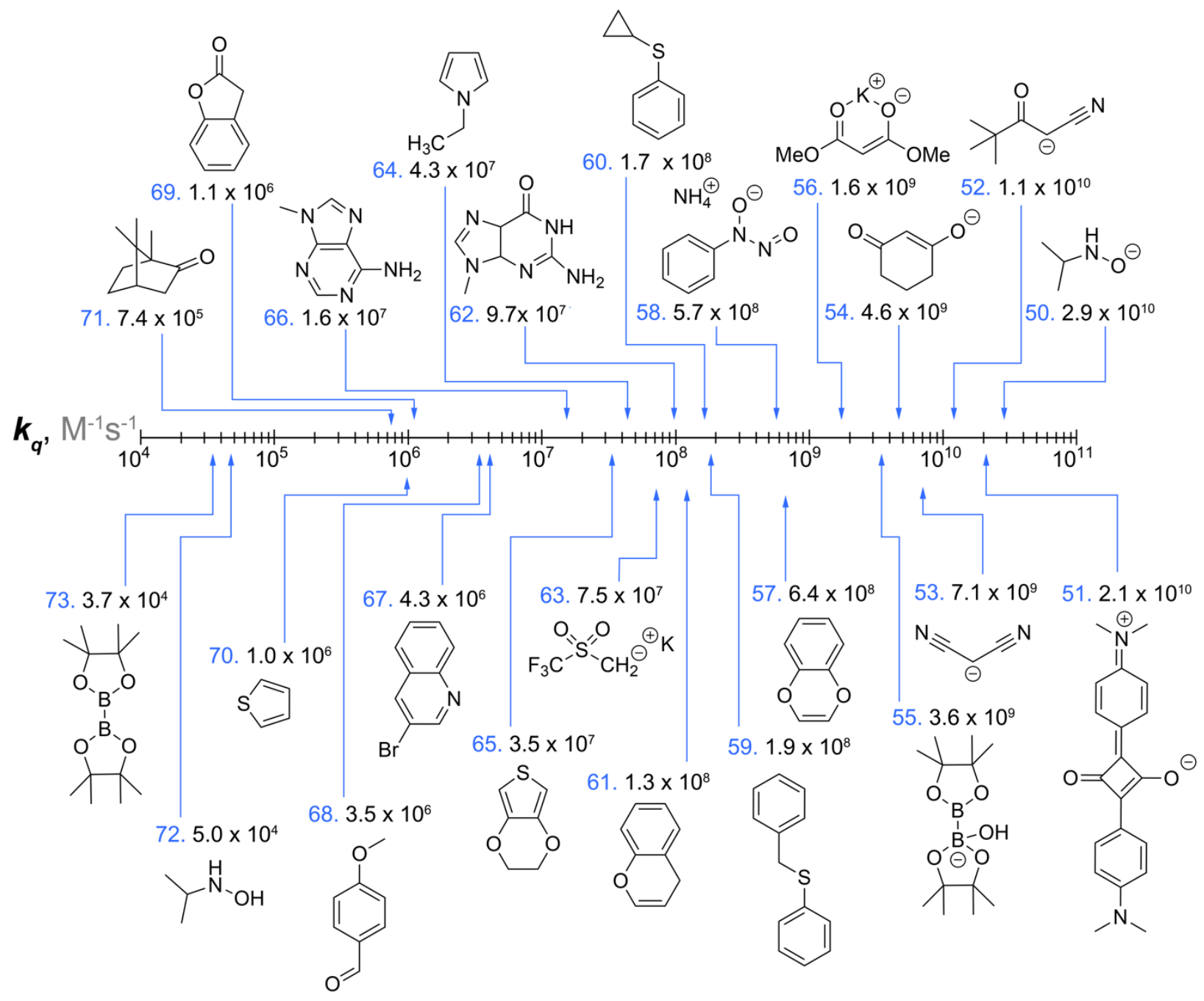

Figure 6. Subset of theoretically predicted bimolecular quenching rate constants $\left(\mathrm{k}_{q}\right)$ for the reaction of ${ }^{*} \mathbf{I r}^{\mathrm{IV}} \mathbf{L}^{\mathbf{0}^{-}}$with various compounds in acetonitrile. See supporting information for the full list of calculated compounds. Molecules with predicted $\log \left(\mathrm{k}_{q}\right)$ of $\sim 7-8$ or more should be considered as potentially useful reagents for synthetic transformations. 


\section{Conclusions}

In many cases, photoredox reactivity can be predicted by using standard reduction potentials. These equilibrium thermodynamic measurements, however, sometimes fail to properly explain the dynamic processes that decide the success or failure of a photoredox reaction.

We measured rate constants for the reaction between 50 quenchers (including $\mathrm{O}_{2}$ ) and a popular iridium-based photocatalyst, $\operatorname{Ir}\left[\mathrm{dF}\left(\mathrm{CF}_{3}\right) \mathrm{ppy}\right]_{2}(\mathrm{dtbpy})^{+}$in acetonitrile. These values could then be used to calibrate a simple method to predict the reactivity of small molecule reagents with this particular photoredox catalyst. The DFTbased predictive tool was able to uncover the reactivity of several unforeseen reagents and we were able to translate the predictions into a successful C-H activation of 2,4-pentanedione as a proof of concept.

\section{ASSOCIATED CONTENT}

Supporting Information. Emission quenching experiments, experimental details, input example for the DFT calculation and interpolation to $\log \left(\mathrm{k}_{\mathrm{q}}\right)$, predicted $\mathrm{k}_{\mathrm{q}}$ for more compounds, DFT calculated structures, model using $\Delta G$ instead of the ionization energy, details about the coupling of acetylacetone with electron rich olefin.

\section{AUTHOR INFORMATION}

\section{Corresponding Author}

* frenette.mathieu@uqam.ca

\section{ACKNOWLEDGMENT}

We gratefully acknowledge NSERC for funding in the form of a Discovery Grant to M.F. (RGPIN-2016-06773) and NSERC scholarships to A.J. and J.M. M.M. and J.M. were research scholars partnered through the RISE Canada program (www.risecanada.ca). We are also grateful to NanoQAM for fluorometer access and to Gaussian and ComputeCanada (www.computecanada.ca) for computational resources.

\section{REFERENCES}

1. Zuo, Z.; Ahneman, D.T.; Chu, L.; Terrett, J.A.; Doyle, A.G.; MacMillan, D.W. Merging photoredox with nickel catalysis: Coupling of $\alpha$-carboxyl sp3-carbons with aryl halides. Science, 2014, 345, 437-440.

2. Yang, H.B.; Feceu, A.; Martin, D.B. Catalyst-Controlled $\mathrm{C}-\mathrm{H}$ Functionalization of Adamantanes using Selective H-Atom Transfer. ACS Cata., 2019, 9, 5708-5715.

3. Oderinde, M.S.; Jones, N.H.; Juneau, A.; Frenette, M.; Aquila, B.; Tentarelli, S.; Robbins, D.W.; Johannes, J.W. Highly Chemoselective Iridium Photoredox and Nickel Catalysis for the Cross-Coupling of Primary Aryl Amines with Aryl Halides. Angew Chem. Int. Ed., 2016, 128, $13413-13417$.

4. Corcoran, E.B.; Pirnot, M.T.; Lin, S.; Dreher, S.D.; DiRocco, D.A.; Davies, I.W.; Buchwald, S.L.; MacMillan, D.W. Aryl amination using ligand-free Ni (II) salts and photoredox catalysis. Science, 2016. 353, 279-283.

5. Terrett, J.A.; Cuthbertson, J.D.; Shurtleff, V.W.; MacMillan, D.W. Switching on elusive organometallic mechanisms with photoredox catalysis. Nature, 2015, 524, 330-334.

6. Oderinde, M.S.; Frenette, M.; Robbins, D.W.; Aquila, B.; and Johannes, J.W. Photoredox mediated nickel catalyzed cross-coupling of thiols with aryl and heteroaryl iodides via thiyl radicals. J. Am. Chem. Soc., 2016, 138,1760-1763.

7. Hu, J.; Wang, J.; Nguyen, T.H.; Zheng, N. The chemistry of amine radical cations produced by visible light photoredox catalysis. Beilstein journal of organic chemistry, 2013, 9, 1977-2001.

8. Franchino, A.; Rinaldi, A.; Dixon, D.J. $\alpha$-Alkylation of ketimines using visible light photoredox catalysis. RSC Adv., 2017, 7, 43655-43659.

9. Shang, T.Y.; Lu, L.H.; Cao, Z.; Liu, Y.; He, W.M.; Yu, B. Recent advances of 1, 2, 3, 5-tetrakis (carbazol-9-yl)-4, 6dicyanobenzene (4CzIPN) in photocatalytic transformations. Chem. Comm., 2019, 55, 5408-5419.

10. Uoyama, H.; Goushi, K.; Shizu, K.; Nomura, H.; Adachi, C. Highly efficient organic light-emitting diodes from delayed fluorescence. Nature, 2012 492, 234-238.

11. Welin, E.R.; Le, C.; Arias-Rotondo, D.M.; McCusker, J.K.; MacMillan, D.W. Photosensitized, energy transfer-mediated organometallic catalysis through electronically excited nickel (II). Science, 2017. 355, 6323, 380-385

12. Roth, H.; Romero, N.; Nicewicz, D. Experimental and calculated electrochemical potentials of common organic molecules for applications to single-electron redox chemistry. Synlett, 2016, 27, 714-723.

13. Jacques, P.; Allonas, X.; Burget, D.; Haselbach, E.; Muller, P. A.; Sergenton, A. C.; Galliker, H. Intramolecular multiple Rehm-Weller plots in photoinduced electron transfer: competition between $\pi$-and $n$-type donor sites in benzylamines. Phys. Chem. Chem. Phys., 1991, 1, 1867-1871.

14. Rehm, D.; Weller, A. Kinetics of fluorescence quenching by electron and $\mathrm{H}$-atom transfer. Isr. J. Chem., 1970, 8 , 259-271.

15. Farid, S.; Dinnocenzo, J.P.; Merkel, P.B.; Young, R.H.; Shukla, D.; Guirado, G. Reexamination of the RehmWeller data set reveals electron transfer quenching that follows a Sandros-Boltzmann dependence on free energy. J. Am. Chem. Soc., 2011; 133, 11580-11587.

16. Fearnley, A.F.; An, J.; Jackson, M.; Lindovska, P.; Denton, R.M. Synthesis of quaternary aryl phosphonium salts: photoredox-mediated phosphine arylation. Chem. Comm., 2016, 52, 4987-4990.

17. Duret, G.; Quinlan, R.; Bisseret, P.; Blanchard, N. Boron chemistry in a new light. Chem. Sci., 2015, 6, 53665382.

18. Isegawa, M.; Neese, F.; Pantazis, D.A. Ionization energies and aqueous redox potentials of organic molecules: comparison of DFT, correlated ab initio theory and pair natural orbital approaches. J. Chem. Theo. and Comp., 2016, 12, 2272-2284.

19. Chai, J.D.; Head-Gordon, M. Systematic optimization of long-range corrected hybrid density functionals. J. Chem. Phys., 2008, 128, 084106.

20. McKechnie, S.; Booth, G.H.; Cohen, A.J.; Cole, J.M. On the accuracy of density functional theory and wave function methods for calculating vertical ionization energies. J. Chem Phys., 2015, 142, 194114.

21. Frisch, M.J.E.A.; Trucks, G.W.; Schlegel, H.B.; Scuseria, G.E.; Robb, M.A.; Cheeseman, J.R.; Scalmani, G.; Barone, V.; Mennucci, B.; Petersson, G.; Nakatsuji, H. 2009. gaussian 09, Revision d. 01, Gaussian. Inc., Wallingford CT, 201

22. Bordwell, F.G.; Cheng, J.P.; Bausch, M.J. Acidities of radical cations derived from remotely substituted and phenyl-substituted fluorenes. J. Am. Chem. Soc., 1988, 110, 2867-2872. 